Relations industrielles

Industrial Relations

\title{
La vie quotidienne des familles ouvrières, par M. Chombart de Lauwe, Centre National de la Recherche Scientifique, Gentilly-Seine, 1959.
}

\section{Laurent Bélanger}

Volume 16, numéro 3, juillet 1961

URI : https://id.erudit.org/iderudit/1021784ar

DOI : https://doi.org/10.7202/1021784ar

Aller au sommaire du numéro

Éditeur(s)

Département des relations industrielles de l’Université Laval

ISSN

0034-379X (imprimé)

1703-8138 (numérique)

Découvrir la revue

Citer ce compte rendu

Bélanger, L. (1961). Compte rendu de [La vie quotidienne des familles ouvrières, par M. Chombart de Lauwe, Centre National de la Recherche Scientifique, Gentilly-Seine, 1959.] Relations industrielles / Industrial Relations, 16(3), 366-367. https://doi.org/10.7202/1021784ar

Tous droits réservés (C Département des relations industrielles de l’Université Laval, 1961
Ce document est protégé par la loi sur le droit d'auteur. L'utilisation des services d'Érudit (y compris la reproduction) est assujettie à sa politique d'utilisation que vous pouvez consulter en ligne.

https://apropos.erudit.org/fr/usagers/politique-dutilisation/ 
au plan de l'objectif ultime, il y a aussi une pluralité sur les plans structurel et idéologique.

La priorité accordée à l'action politique plutôt qu'à la négociation collective explique pour une part la centralisation ou la décentralisation du pouvoir au sein des mouvements. La forme de propriété de l'entreprise rend compte aussi de la centralisation du pouvoir. Nationalisée ou entre les mains de capitaux privés, la propriété demeure centralisée. Le pluralisme idéologique et structurel ne peut être expliqué par la nature de l'industrie, puisque l'idéal politique ne conduit pas nécessairement au pluralisme syndical. La Grande-Bretagne illustre ce fait. $\mathrm{La}$ variable décisive dans l'explication du pluralisme serait la suivante: «The crucial determinants of union structure is the view that a national labor movement or a national working class takes of its own role».

Cette analyse constitue un document important pour ceux qui étudient le syndicalisme avec l'approche structurellefonctionnelle ou encore pour ceux qui s'intéressent aux problèmes de démocratie syndicale.

\section{LAURENT BÉLANGER}

La vie quotidienne des familles ouvrières, par M. Chombart de Lauwe, Centre National de la Recherche Scientifique, Letouzey et Ané, GentillySeine, 1959.

Cette étude empirique sur la vie des familles ouvrières est un exposé des relations entre les conditions de vie de ces familles et leurs comportements sociaux, en particulier leur comportement alimentaire. L'interprétation des données tient compte toujours du milieu social, i.e. «de l'ensemble des facteurs sociaux se trouvant en relation avec les comportements et les personnalités des sujets et avec la forme et comportement des groupes 》.

L'orientation générale de la recherche est de dépasser la description de la situation et l'interprétation quantitative des rapports entre les deux grandes variables, pour découvrir les facteurs psychosociologiques qui sous-tendent les comportements sociaux. Ces facteurs sont les réactions des familles à l'endroit de leurs conditions de vie, leurs attitudes, leurs aspirations, leurs motivations, leurs coutumes, etc.

L'enquête, portant sur les travailleurs salariés et leur famille habitant les quartiers de Paris et de la banlieue, commen. ce en 1950. C'est la reprise économique après une récession. La conjoncture économique est un facteur dont il faut tenir compte dans l'interprétation des données puisque l'intérêt * tient au fait que les familles ouvrières sont le récepteur le plus sensible aux perturbations économiques et aux déséquilibres sociaux 》.

Pour préciser le concept de famille ouvrière, correspondant à classe ouvrière, l'auteur établit des catégories et souscatégories socio-professionnelles. L'interprétation des rapports entre conditions de vie et comportements montre les variations sensibles suivant la classe et les catégories professionnelles au sein de la classe ouvrière.

La variable «conditions de vie » comprend les éléments suivants: le travail de l'homme et ses conditions d'existence, le travail de la femme et l'équilibre du ménage, les conditions de logement et l'espace familial.

Au chapitre du travail de l'homme, l'auteur souligne l'opposition entre le milieu de travail et le milieu résidentiel: le premier est un milieu où l'activité est orienté vers la production, l'adaptation aux techniques nouvelles; le second est un milieu de consommation, où dominent les valeurs traditionnelles, les relations familiales et de voisinage. L'emploi, le métier et la vie syndicale ont des répercussions sur la vie familiale.

Le travail de la femme impose un rythme à la vie de famille. Ce dernier, en retour, influe sur le rendement dans l'entreprise. Ce travail modifie les rapports entre parents et enfants, les habitudes de consommation par l'apport d'un revenu accru.

L'étude sur les logements se centre sur les points suivants: ce qu'expriment les logements et conditionnements qu'ils imposent; ce que devraient être les logements. L'auteur arrive à la détermination d'un seuil critique au-dessous duquel les maladies physiques ou mentales peuvent apparaître. Il traite du décalage entre les désirs et les conditions existantes, des types de rapports sociaux entre familles ouvrières et familles bourgeoises. 
Le comportement social est \& un ensemble de pratiques et d'attitudes prédominantes chez les individus d'un même groupe liés à des besoins et des aspirations ». L'enquête porte ici sur les budgets et les pratiques de consommation: pratiques d'achat, organisation des dépenses et du crédit, rôle de l'homme et de la femme dans l'administration financière du ménage. L'auteur reprend les études sur le minimum vital et les budgets-types. L'étude sur le comportement alimentaire se centre autour de la consommation de la viande. Les conclusions particulières sont les suivantes:

«En fonction de leurs conditions de vie, de leur dépense physique et de leurs revenus, la moyenne des ouvriers avaient une consommation alimentaire proportionnellement plus faible que celle des autres classes. 》

« La consommation de la viande ne correspondrait pas directement à la satisfaction d'un besoin métabolique. >

En traitant des motivations et attitudes qui sous-tendent les comportements, l'auteur précise deux types de comportement: le comportement de préoccupation et celui d'intérêt libre. Cette distinction l'amène à élargir le concept de besoin: on distingue besoins physiques et besoins phychologiques. Ces derniers se subdivisent en abesoins-obligation 》, «besoins-détente», «besoins-aspiration».
D'une façon globale, l'étude des comportements montre que les ménages ouvriers sont « les plus soumis matériellement aux fluctuations de la conjoncture ». \& Les pourcentages des chapitres des budgets varient suivant les saisons de l'année et les crises économiques. Les rythmes de la vie économique du ménage sont en relation avec l'aménagement de l'espace, avec différentes phases de l'existence et surtout avec dates de paiement des salaires \$.

Ce travail de recherche tire son intérêt réel non seulement de l'exposé des résultats obtenus, mais aussi de sa contribution théorique et méthodologique en sociologie de la famille et du logement.

La détermination des seuils critiques et optima, le démontage du mécanisme d'un comportement social, la recherche d'un degré de signification des résultats statistiques, la précision des concepts: espace social objectif, espace social subjectif, milieu social, etc., constituent un apport théorique qui mérite d'être considéré. La détermination géographique de l'enquête, l'échantillonage soit au hasard ou par choix raisonné, la construction du questionnaire d'attitudes, la conduite des interviews sont autant de techniques utilisées avec doigté au cours de la recherche.

\section{LAURENT BÉLANGER}

\section{PUBLICATIONS RÉCENTES RECENT PUBLICATIONS}

\section{Organisation de l'entreprise}

«La décision d'automatiser la gestion et ses conséquences », par M. Pèpe, Gestion Organisation, 92, rue Bonaparte, Paris 6e, mars 1961, pp. 93-96.

«The Plannig Function in the Business Enterprise \&, by Norman F. Dufty, Journal of the Academy of Management, Michigan State University, East Lansing, Volume 4, Number 1, April 1961, pp. 51-58.

«A Suggested Solution to Line Versus
Staff », by Fred E. Kindig, Journal of the Academy of Management, Michigan State University, East Lansing, Volume 4, Number 1, April 1961, pp. 59-61.

« La recherche opérationnelle au service de l'entreprise », Numéro spécial, Gestion Organisation, 92, rue Bonaparte, Paris 6e, avril 1961, pp. 125-184.

«La fonction de la direction dans l'industrie et le commerce », par Sir Frédéric Hooper, Management International, Graduate School of Business, Administra- 